\title{
Gastric cancer in Korea
}

\author{
Hyuk-Joon Lee ${ }^{1}$, Han-Kwang Yang ${ }^{1}$, and Yoon-OK Ahn ${ }^{2}$ \\ ${ }^{1}$ Department of Surgery and Cancer Research Institute, Seoul National University College of Medicine, 28 Yongon-Dong, Chongno-Gu, \\ Seoul 110-744, Korea \\ ${ }^{2}$ Department of Preventive Medicine, Seoul National University College of Medicine, Seoul, Korea
}

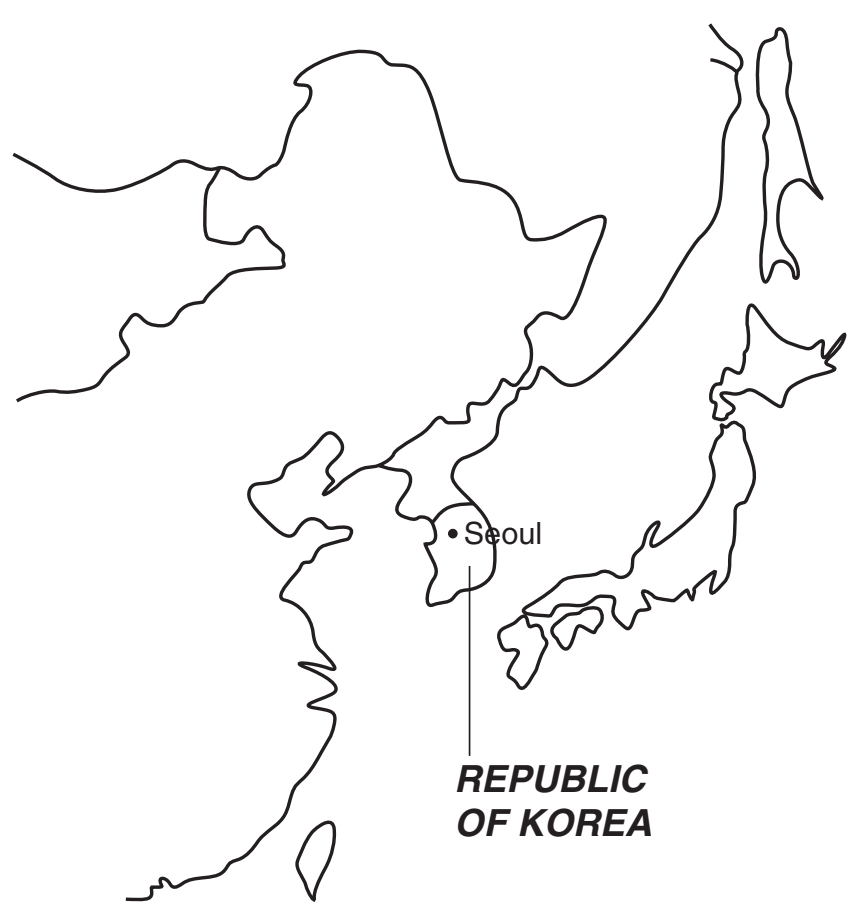

\section{Introduction}

Although the incidence and mortality of gastric cancer have been decreasing, it is still the second most common cancer, next to lung cancer, around the world today [1]. Korea is an area that has one of the highest incidence rates of gastric cancer in the world, especially for men [2].

Offprint requests to: H.-K. Yang

Received: April 3, 2002 / Accepted: July 2, 2002

\section{Cancer statistics in Korea}

According to the Seoul cancer registry data, the crude annual incidence rate of all sites of cancer per 100000 population was 187.6 for males and 164.3 for females in 1993-1997 (Table 1). The age-standardized rate for the world population was 284.0 in males and 172.3 in females.

Cancer was the third or fourth most common cause of death until the 1970s and had become the second in the 1980s. In 2000, cancer became the leading cause of death in Korea (Fig. 1), accounting for $23.9 \%$ of all deaths in 2000 ( $27.5 \%$ for males and $19.4 \%$ for females). From 1990 to 2000, cerebrovascular diseases, heart diseases, and liver diseases were decreasing, but the overall cancer mortality rate increased by $9.7 \%$ [3]. The crude annual death rate of all cancers was 157.9 per 100000 for males and 90.2 for females, and the age-standardized death rate of all cancers was 184.2 per 100000 for males and 74.9 for females in 2000 (Table 1).

\section{Gastric cancer incidence in Korea}

Gastric cancer is the most prevalent malignant neoplasm in Korea, comprising $20.8 \%$ of all cancer sites. For both sexes, gastric cancer is the leading malignancy, although it is less predominant in women $(15.8 \%)$ than in men $(24.5 \%)$ (Figs. 2, 3) [4]. The age-standardized incidence rate of gastric cancer was 68.1 per 100000 for males and 29.0 per 100000 for females annually from 1993 to 1997, as reported by the Seoul Cancer registry.

The incidence rate of gastric cancer per 100000 per year in Korea is less than 1.0 in children and adolescents younger than age 20 years. Its incidence rate starts to rise in middle-aged adults. In men, the incidence rate of gastric cancer is doubled, with 5-year intervals, from age 45 to 60 years, and is increased by $50 \%$ with 5 -year intervals thereafter. The incidence peaks in males be- 
Table 1. Cancer incidence and mortality statistics, Korea, 2000

\begin{tabular}{lccc}
\hline Index & Males & Females & Total \\
\hline Annual incidence rate (per 100000) & & & \\
$\quad$ Crude rate & 187.6 & 164.3 & 175.9 \\
$\quad$ Age-standardized rate & 284.0 & 172.3 & 214.8 \\
Annual death rate (per 100000) & & & \\
$\quad$ Crude rate & 157.9 & 90.2 & 124.1 \\
$\quad$ Age-standardized rate & 184.2 & 74.9 & 120.3 \\
Relative frequency of cancer death & & & 23.9 \\
$\quad$ Percentage of all deaths & 27.5 & 19.4 & 1 st \\
$\quad$ Order in cause of death & 1 st & 1 st &
\end{tabular}

National Statistical Office of Korea. Annual Report on the Cause of Death Statistics, 2000

(per 100,000)

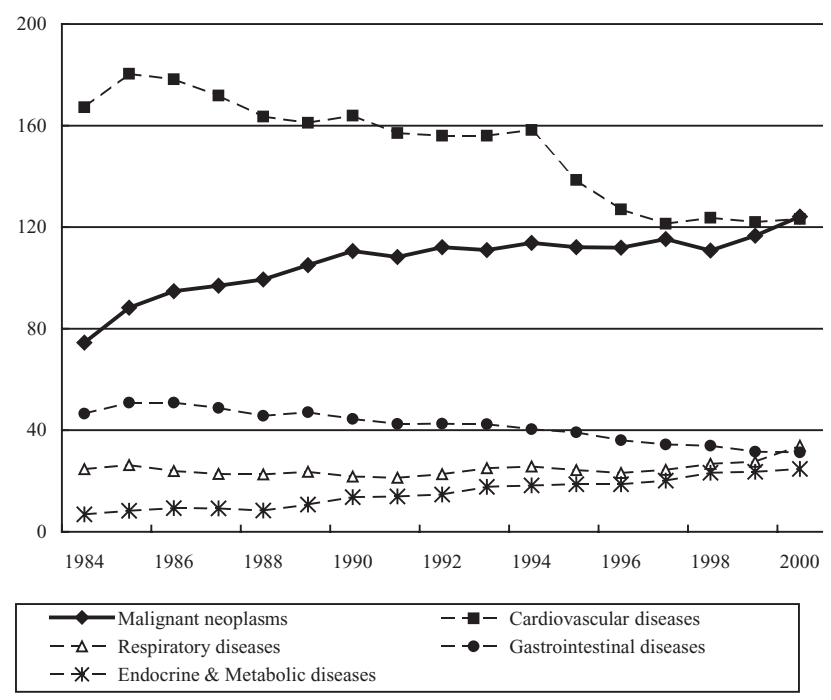

National Statistical Office, Annual report on the cause of death statistics, 1984-2000

Fig. 1. Annual mortality trends by leading causes of death in Korea, 1984-2000

tween ages 75 and 79 years; in this group, it approaches about 600 per 100000 . This trend for gastric cancer is similar in females, and its peak incidence is about 230 per 100000 between ages 75 and 79 years. In both men and women, the incidence decreases after age 80 years. The cumulative risk of gastric cancer for the age span 0 74 years in Korea reaches $9.0 \%$ in males and $3.7 \%$ in females [5].

In detail, the age incidence patterns by sex show a female preponderance in those under 35 years of age and reverse to a male preponderance in those over this age (Fig. 4). This cross phenomenon of the gastric cancer incidence curve between the sexes, which was also noted among the Japanese in Osaka [6], implies that the etiologic nature or major risk factors for gastric cancer affecting the younger age groups may be different from those affecting the older age groups.

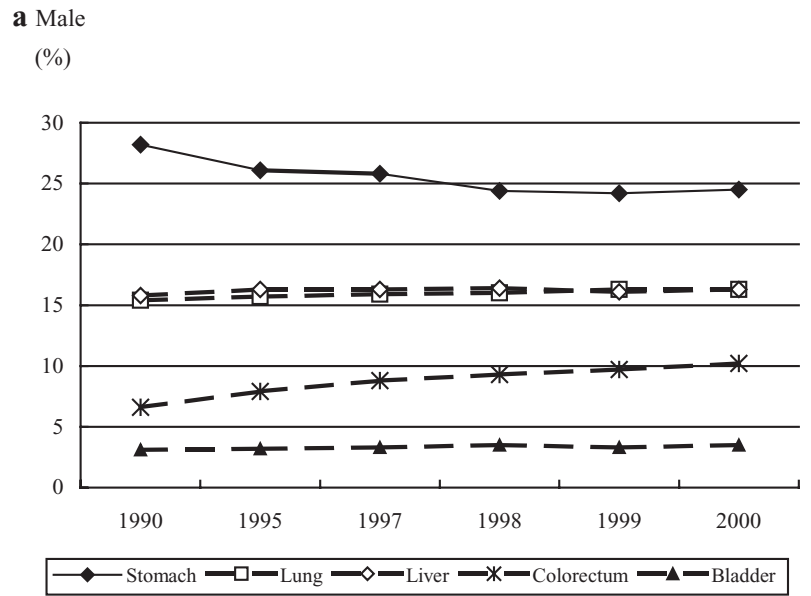

b Female

(\%)

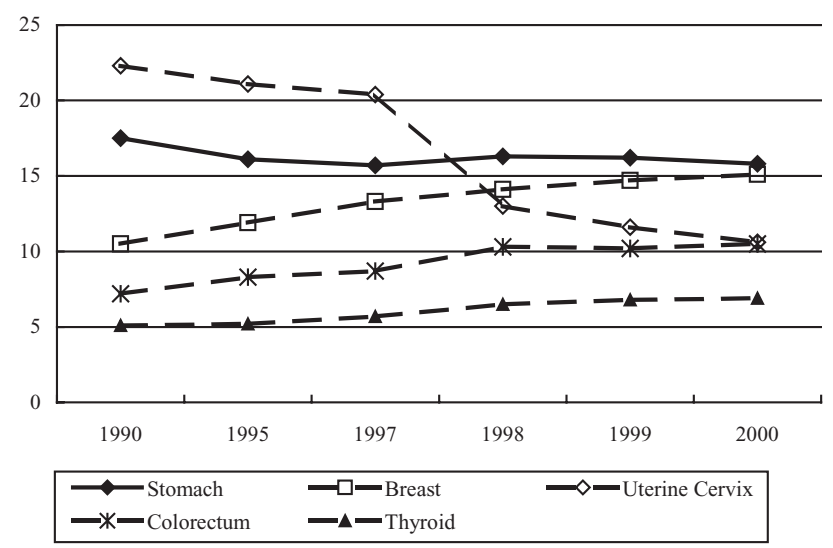

Central cancer registry in Korea, Annual report of the central cancer registry in Korea, 1990-2000

Fig. 2a,b. Annual relative proportions of major cancer incidences by sex. a Male; $\mathbf{b}$ female 
Korea and some parts of eastern Asia, including Japan, have the highest incidence of gastric cancer in the world [2]. The age incidence curves for males in Seoul, Korea; Osaka, Japan; and for white males in Los Angeles, the United States are shown in Fig. 5. The curve for Seoul parallels that for Los Angeles; in other words, it has same the difference ratios, about 9.3-9.4, across the age groups. This parallel suggests that additional or particular factors, which are common across the age groups, are involved in the risk of gastric cancer in Korea. However, the curve for Osaka is not parallel to

(a) Male
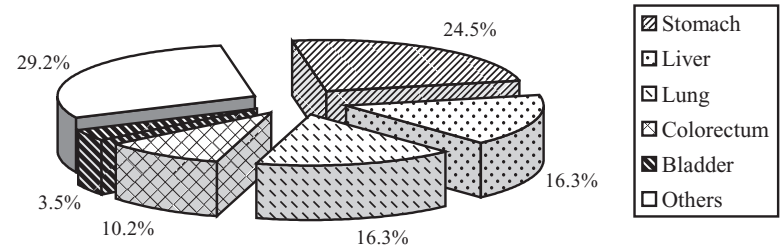

(b) Female

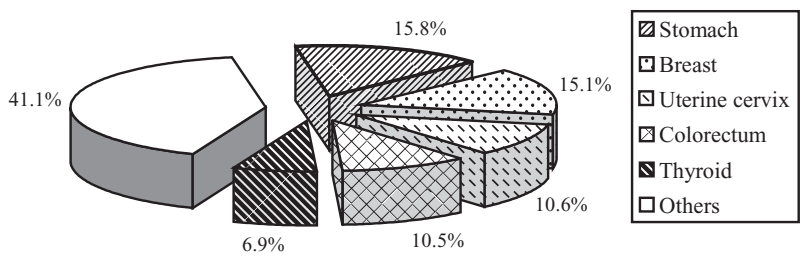

Central cancer registry in Korea, Annual report of the central cancer registry in Korea, 2000

Fig. 3a,b. Relative frequencies of the primary sites of cancer in Korea in 2000. a Male; b female that for Seoul along all the age groups. In those under the age of 40 years, the incidence in Osaka is relatively reduced. This suggests that the risk of gastric cancer is changing in Japan, especially in the younger population.

\section{Gastric cancer mortality in Korea}

In Korea, the crude death rate for gastric cancer is 24.3 per 100000 , which is the second leading cause of cancer death, slightly lower than the rate for lung cancer (24.4 per 100000). In females, gastric cancer is the leading cause of cancer death, with a crude death rate of 17.2 per 100000, but in males, it is the third leading cause of cancer death (31.3 per 100000), next to lung and liver cancer (36.1 and 32.5 per 100000 , respectively) [3]. The age-standardized death rate for gastric cancer in Korea is 36.7 per 100000 in males, and 13.9 in females.

(per 100,000)

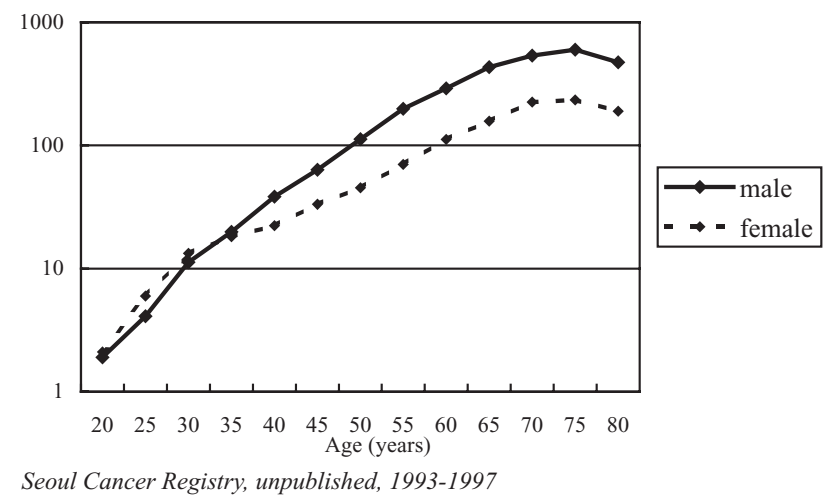

Fig. 4. Age-specific incidence curves of gastric cancer in Korea by sex, 1993-1997

(per 100,000)

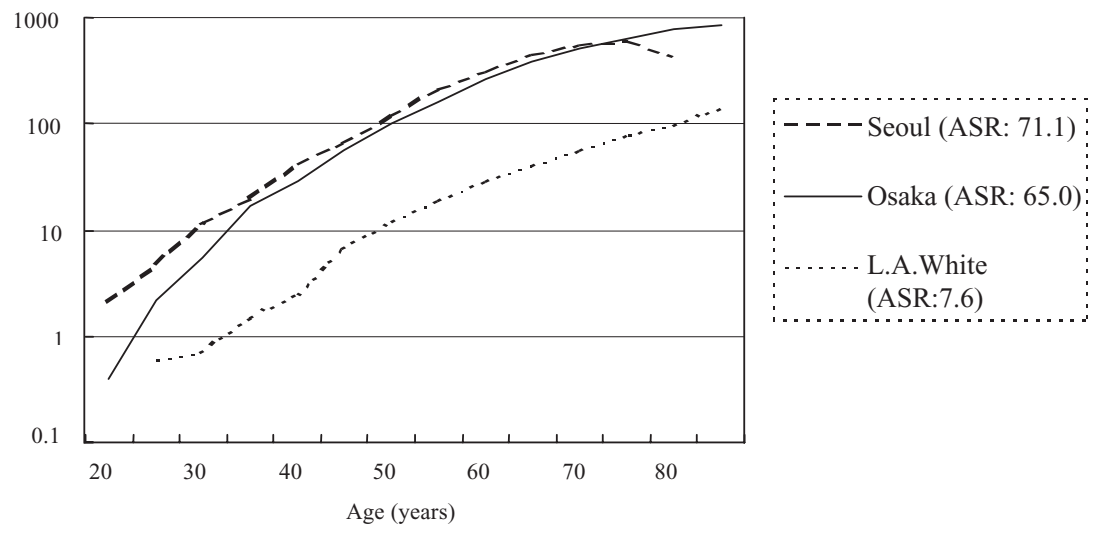

Fig. 5. Incidence curves of stomach cancer by age among males in Seoul (19921995), Osaka (1988-1992), and Los Angeles (L.A.); Whites (1988-1992). $A S R$, Age-standardized rate 
As shown in Fig. 6, the death rate for gastric cancer in Korea has been decreasing slightly over the past 20 years, although it is still high. Interestingly, this decreasing trend was marked in the middle-aged group (aged 35-64 years), but not in the older group (Fig. 7).

\section{Risk factors}

For gastric cancer, a wide variety of etiologic risk factors, including dietary factors, chronic atrophic gastritis, intestinal metaplasia, and Helicobacter pylori infection, have been investigated. However, none of the epidemiologic factors have been sufficient to account for the pathogenesis of gastric cancer [7].

Approximately $10 \%$ of gastric cancers show familial clustering, and case-control studies have identified a three fold increased risk of the disease in the first-

(per 100,000)

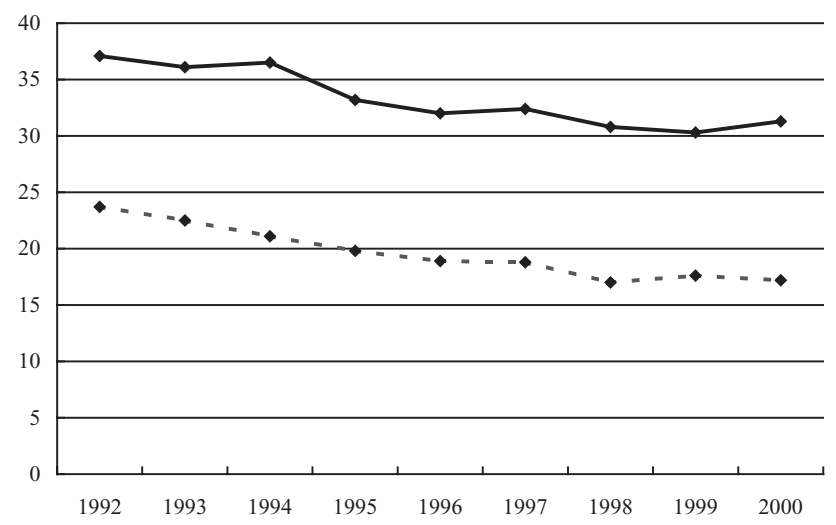

National Statistical Office, Annual report on the cause of death statistics, 1992-2000

Fig. 6. Crude mortality rates of gastric cancer by sex, 19922000. Symbols, As in Fig. 4 degree relatives of affected individuals [8]. According to the criteria for hereditary gastric cancer presented by the International Gastric Cancer Linkage Consortium (IGCLC) in 1999, only 1\% of gastric cancers meet the criteria [9]. Similarly, in Korea, the relative risk in the first-degree relatives of gastric cancer patients is 3.1, compared with the general population [10]. The ratio of hereditary gastric cancer, according to the criteria of the IGCLC, is $0.3 \%-3.1 \%$ of all gastric cancers [11].

Gastric cancer is known as the second most common extracolonic cancer, next to endometrial cancer, in cases of hereditary nonpolyposis colorectal cancer (HNPCC). In Korea, the calculated risk of gastric cancer in patients with HNPCC and their first-degree relatives was 2.1-fold greater than that in the general population. In addition, the increased risk was particularly marked at a younger age; the relative risk was increased up to 11.3-fold in subjects in their thirties and 5.5-fold in those in their forties [12].

Dietary factors are believed to be one of the most important risk factors for gastric cancer. The dietary habits of Korean people can be characterized as a high intake of salty foods and carbohydrates and a higher intake of cooked than fresh vegetables. Several casecontrol studies showed that an increased risk of gastric cancer was noted among those with a high consumption of stewed foods, such as soybean paste stew and hot pepper-soybean stew, broiled fish, and those who liked salty food. Analysis of cooking methods showed that broiling and salting increased the risk of gastric cancer, but that frying tended to decrease the risk $[10,13,14]$.

Helicobacter pylori infection has been accepted as a class I carcinogen for the development of gastric cancer, although a definite causal model does not exist. The seroprevalence of $H$. pylori infection in Korean adults has decreased from $80 \%$ in the 1980 s to the $67 \%$ in the 1990s [15]. Previous reports from areas with a high inci- (per 100,000)

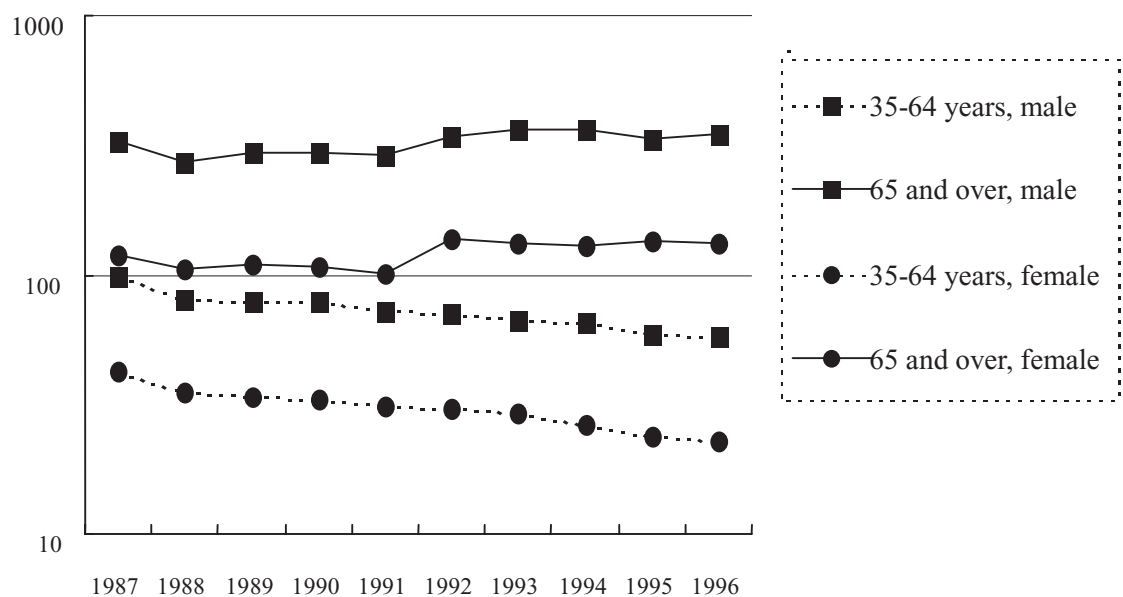

Fig. 7. Trends in gastric cancer mortality rates by age group: Korea, 1987-1996 
dence of gastric cancer, such as Korea and China, have failed to document any relationship between $H$. pylori infection and gastric cancer. But, recently, one casecontrol study revealed that $H$. pylori infection was one of the important risk factors for the development of gastric cancer in Korea, with an odds ratio of 1.82 [16].

Other factors contributing to the development of gastric cancer in Korea are cigarette smoking (odds ratio, 3.4 ) and the use of a refrigerator (odds ratio, 0.64) $[10,14]$.

\section{Pathologic features}

Adenocarcinomas account for $95.8 \%$ of all gastric malignancies in Korea. The percentages of signet-ring cell carcinoma and mucinous carcinoma are $16.8 \%$ and $1.7 \%$, respectively, in gastric adenocarcinomas [4].

According to the recent report of the Korean Gastric Cancer Association [17], the proportion of early gastric cancer (EGC) had increased from $28.6 \%$ among 5380 operated patients in 1995 to $32.8 \%$ among 6772 patients in 1999. The International Union Against Cancer (UICC) staging showed $29.3 \%$ with stage Ia, $13.9 \%$ with stage Ib, $14.8 \%$ with stage II, $13.2 \%$ with stage IIIa, $6.3 \%$ with stage IIIb, and $18.1 \%$ with stage IV in 1999. In early gastric cancer, type IIc was the most common $(42.8 \%)$. The incidence of upper-one-third cancer increased slightly in 1999 (12.5\%) compared with 1995 $(11.2 \%)$, and this was reflected in the increased rate of proximal gastrectomy in 1999 (207 cases; 3.6\%).

\section{Treatment and results}

Most gastric cancer patients in Korea receive surgery alone $(42.8 \%)$ or surgery with adjuvant chemotherapy $(17.4 \%)$ [4]. Operations for gastric cancer patients in 1999 were: subtotal gastrectomy $(67.3 \%)$, total gastrectomy $(24.1 \%)$, proximal gastrectomy $(3.6 \%)$, wedge resection $(0.5 \%)$, bypass surgery $(1.8 \%)$, and open biopsy $(2.7 \%)$ [17].

According to the database of the Seoul National University Hospital, from 1986 to 1995, the 5-year survival rate of their 6314 patients who received operative treatment was $55.6 \%$. According to the TNM stage, the 5year disease-free survival rates of patients with stages Ia, Ib, II, IIIa, IIIb, and IV were $98.0 \%, 89.7 \%, 71.6 \%$, $53.9 \%, 36.5 \%$, and $23.9 \%$, respectively (Fig. 8).

In Korea, most surgeons perform extended lymph node dissection (D2 or D3) rather than limited lymph node dissection (D1). The number of lymph nodes resected (mean \pm SD) during gastrectomy in Korea was $35.9 \pm 18.5$ in $1999,29.4 \pm 16.6$ in the hospitals where fewer than 100 gastrectomies were performed yearly

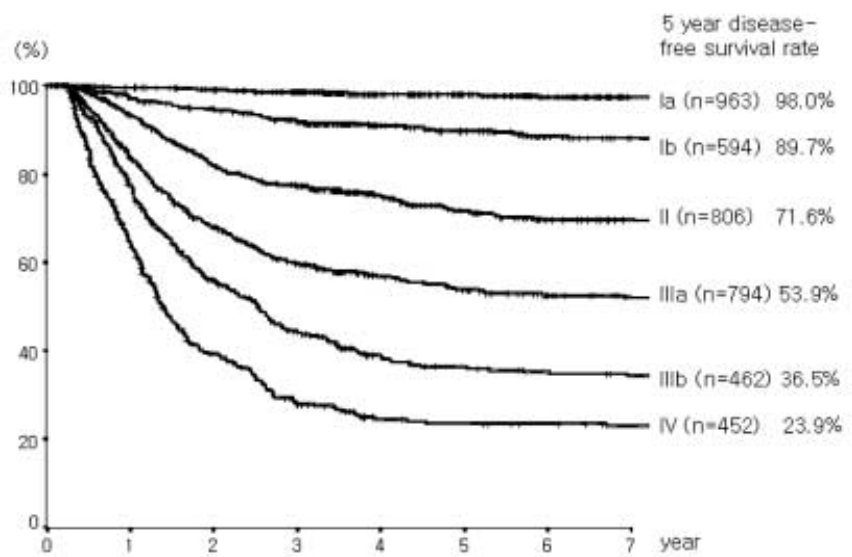

Fig. 8. Five-year disease-free survival curves after curative resection of gastric cancer according to TNM stage; database of Seoul National University Hospital (1986-1995)

and $41.8 \pm 18.3$ in the hospitals where more than 500 gastrectomies were performed yearly [17].

Regarding chemotherapy for gastric cancer, a few prospective randomized phase III trial were performed. According to a clinical trial of 5-fluorouracil (5-FU) and cisplatin (FP) versus 5-FU, doxorubicin, and mitomycin $\mathrm{C}$ (FAM) versus 5-FU alone (FU) in previously untreated patients with advanced gastric cancer, the FP regimen showed a significantly higher response rate than the FAM or FU regimens ( $51 \%$ for FP; $25 \%$ for FAM; $26 \%$ for FU), but did not show a survival benefit [18]. A few multi-institutional prospective, randomized clinical trials of adjuvant chemotherapy for gastric cancer are ongoing in Korea.

\section{References}

1. Cheung LY, Delcore R. Gastric cancer. In: Townsend CM, editor. Sabiston textbook of surgery. 16th Ed. Philadelphia: WB Saunders; 2001. pp 855-65.

2. National Cancer Institute. Stomach - U.S. Racial/ethnic cancer patterns. http://www.nci.nih.gov/statistics

3. National Statistical Office. Annual report on the cause of death statistics (based on vital registration). Seoul: NSO; 2000.

4. Central Cancer Registry in Korea. Annual report of the Central Cancer Registry in Korea. Seoul: Ministry of Health and Welfare, Republic of Korea; 2000.

5. Ahn YO. Epidemiology of gastric cancer. In: Kim JP, editor. Gastric cancer (in Korean). Seoul: Eui-Hak; 1999. pp 1-8.

6. International Agency for Research on Cancer. Cancer incidence in five continents, vol. V. Lyon: IARC Scientific Publication no. 88; 1987.

7. Stadtländer CT, Waterbor JW. Molecular epidemiology, pathogenesis and prevention of gastric cancer. Carcinogenesis 1999; 20:2195-208.

8. Zanghieri G, Di Gregorio C, Sacchetti C, Fante R, Sassatelli R, Cannizzo G, et al. Familial occurrence of gastric cancer in the 2-year experience of a population-based registry. Cancer 1990; 66:2047-51 
9. Shinmura K, Kohno T, Takahashi M, Sasaki A, Ochiai A, Guilford P, et al. Familial gastric cancer: clinicopathological characteristics, RER phenotype and germline $p 53$ and E-cadherin mutations. Carcinogenesis 1999;20:1127-31.

10. Ahn YO. Diet and stomach cancer in Korea. Int J Cancer 1997;(Suppl)10:7-9.

11. Kim SJ, Cho SJ, Heo SC, Yang HK, Kim WH, Park JG, et al. The incidence of hereditary gastric cancer in Korean. J Korean Cancer Assoc 2000;32:1-6.

12. Park YJ, Shin KH, Park JG. Risk of gastric cancer in hereditary nonpolyposis colorectal cancer in Korea. Clin Cancer Res 2000; 6:2994-8.

13. Lee JK, Park BJ, Yoo KY, Ahn YO. Dietary factors and stomach cancer: a case-control study in Korea. Int J Epidemiol 1995;24:3341.

14. Kim HJ, Chang WK, Kim MK, Lee SS, Choi BY. Dietary factors and gastric cancer in Korea: a case-control study. Int J Cancer 2002;97:531-5.

15. Kim HS, Lee YC, Lee HW, Yoo HM, Lee CG, Kim JM, et al. Seroepidemiologic study of Helicobacter pylori infection in Korea. Korean J Gastroenterol 1999;33:170-82.

16. Chang WK, Kim HY, Kim DJ, Lee J, Park CK, Yoo JY, et al. Association between Helicobacter pylori infection and the risk of gastric cancer in the Korean population: prospective casecontrolled study. J Gastroenterol 2001;36:816-22.

17. Korean Gastric Cancer Association. Nationwide gastric cancer report in Korea. J Korean Gastric Cancer Assoc 2002;2:105-14.

18. Kim NK, Park YS, Heo DS, Suh C, Kim SY, Park KC, et al. A phase III randomized study of 5-fluorouracil and cisplatin versus 5-fluorouracil, doxorubicin, and mitomycin $\mathrm{C}$ versus 5 fluorouracil alone in the treatment of advanced gastric cancer. Cancer 1993;71:3813-8. 